hypomethylation, $\mathrm{p}=0.009)$ despite modest but significant upregulation of expression (0.24, $\mathrm{p}=8.20 \mathrm{E}-03)$.

Conclusions Our findings could be the first to suggest that the up- and downregulation of BAG3 and EIF6 expression in MC could be due to hypo- and hypermethylation, however, their specific roles in MC tumourigenesis remains to be elucidated. Beyond mucin genes, upregulation of genes rarely associated with CRC was also observed, suggesting new insight into the aetiology of $\mathrm{MC}$ resistance to therapies and their roles as a potential target for $\mathrm{MC}$ treatment may be worth investigating.

\section{IDDF2018-ABS-0243 TSG-6 RELEASED BY MESENCHYMAL STEM CELLS DERIVED FROM HUMAN INDUCED PLURIPOTENT STEM CELLS ALLEVIATE COLITIS THROUGH THE REGULATION OF CD44}

Hongsheng Yang*, Rui Feng, Shenghong Zhang, Shu Xu, Yun Qiu, Ting Feng. Department of Gastroenterology, The First Affiliated Hospital of Sun Yet-sen University, China

\subsection{6/gutjnl-2018-IDDFabstracts.40}

Background Mesenchymal stem cells (MSCs) have become a promising approach for inflammatory bowel disease (IBD). MSCs derived from human induced pluripotent stem cells (iPSC-MSCs) have increased expandability and prolonged stemness, but the therapeutic effects need to be further investigated. Tumour Necrosis Factor-stimulated Gene-6 (TSG6) released from MSCs have been shown to accelerate intestinal repair in IBD. And only CD44 acts as a TSG6 receptor. Therefore, we want to investigate the potential role and mechanism of the iPSC-MSCs on murine colitis via the regulation of TSG-6 and CD44.

Methods Murine colitis was induced by intrarectal TNBS administration. The expression of TSG6 in iPSC-MSCs was assayed with immunocytochemistry and Western blot. To study the role of TSG-6 secreted by iPSC-MSCs in alleviating murine colitis, iPSC-MSCs $\left(2 \times 10^{6}\right.$ cells/mouse $)$ transfected with or without siRNA targeting TSG6 were injected intraperitoneally on the next day after TNBS administration. Body weight and disease activity index were recorded daily. Cell proliferation, apoptosis and the expression of CD44 were analysed by immunohistochemistry or qRT-PCR. In addition, the role of CD44 was studied by applying either control peptide or CD44 signalling inhibitor, PEP-1 to TNBS-induced murine colitis. Mice symptoms and mucosal healing were analysed as also.

Results iPSC-MSC produced and released TSG6. And the injection of iPS-MSC intraperitoneally attenuated murine colitis accompanied by increasing body weight and reducing the size of the ulcer. However, the therapeutic effects were largely decreased by knockdown of TSG6 in iPSC-MSC. In addition, iPS-MSC accelerated the ulcer healing by promoting the proliferation (Ki67) of colonic epithelial cells and increasing expression of CD44, but not in mice given iPSC-MCS with TSG-6 knockdown. And then we assessed the possible mechanism of TSG6 in promoting mucosal healing by CD44, and we found that the therapeutic effects of TSG-6 itself were similar to iPS-MSC. But the protecting effects of TSG-6 were diminished by inhibition of CD44 via injection of PEP-1 to TNBS induced colitis mice.

Conclusions iPSC-MSCs alleviate murine colitis by releasing TSG-6 and then activating CD44.
IDDF2018-ABS-0244 SCREENING OF CHROMOSOMAL ALTERATIONS, K-RAS MUTATIONS AND DNA METHYLATION STATUS IN APC PROMOTER OF FAMILIAL AND SPORADIC CASES WITH CARCINOMA OF THE AMPULLA OF YATER IN TAMIL NADU POPULATION

${ }^{1}$ Balachandar Vellingiri ${ }^{*},{ }^{2}$ Anand Lakshmanan, ${ }^{1}$ Venkatesh Balasubramanian, ${ }^{3}$ Kaavya Jayaramayya, ${ }^{3}$ Mahalaxmi Iyer, ${ }^{4}$ Mohana Devi Subramaniam. ${ }^{1}$ Human Molecular Genetics and Stem Cells Laboratory, Department of Human Genetics and Molecular Biology, Bharathiar University, Coimbatore, Tamil Nadu, India; Institute of Surgical Gastroenterology, Government Stanley Medical College Hospital, Chennai, India; ${ }^{3}$ Department of Zoology, Avinashilingam Institute for Home Science and Higher Education for Women - Avinashilingam University for Women, Coimbatore, Tamil Nadu, India; ${ }^{4}$ Department of Genetics and Molecular Biology, Vision Research Foundation, Sankara Nethralaya, India

\subsection{6/gutjnl-2018-IDDFabstracts.41}

Background Carcinoma of the ampulla of Vater is a rare malignant tumour arising from the precancerous lesions, which accounts for $6 \%-7 \%$ of periampullary tumours and $0.2 \%$ of gastrointestinal tract malignancies. The aim of the study was to identify the chromosomal alterations and K-ras mutations and to check the methylation status in Adenomatous polyposis coli (APC) promoter region in the familial and sporadic carcinomas of the ampulla of Vater.

Methods A total of 42 samples of carcinoma of the ampulla of Vater (included 31 familial and 11 sporadic cases) and equal numbers of controls were selected which were categorised based on their age (group $\mathrm{I}<50$ years; group II $>50$ years). Techniques including GTG-banding and PCR-RFLP were used to identify the genetic alterations. DNA methylation in APC promoter region was analysed for methylation status at the $\mathrm{CpG}$ regions.

Results The result revealed a high frequency of chromosomes $1 p-$ and $12 p+$ involved in the poorly differentiated (PD) tumour grade and an increased prevalence of the K-ras mutations at the codon 12 associated with $>2 \mathrm{~cm}$ tumour size in the familial carcinomas of the ampulla of Vater. The APC promoter region showed hypermethylation in the $\mathrm{CpG}$ islands of carcinoma patients compared to controls.

Conclusions The results concluded that the chromosomes $1 \mathrm{p}$ and $12 p+$ region might play a vital role for the development of a high grade tumour and the K-ras gene mutation is an early molecular event leading to an abnormal proliferation of the cells. Methylation profiling showed that APC gene has a key role in stabilising b-catenin pathway, in which hypermethylation in APC promoter region could lead to proteasome degradation of b-catenin suggesting that APC methylation may be the potential testing for Carcinoma of the ampulla of Vater diagnosis and provide a new viewpoint in the treatment.

\section{IDDF2018-ABS-0249 SPONTANEOUS CHOLECYSTOCOLONIC FISTULA IN AN ELDERLY MALE: A RARE CAUSE OF MASSIVE LOWER GI BLEEDING}

Millette Castro*. University of the East Ramon Magsaysay Memorial Medical Center, Philippines

10.1136/gutjnl-2018-IDDFabstracts.42

Background Biliary enteric fistulas are often incidental findings. When asymptomatic usually no treatment is necessary 
but when they present with significant complication, surgery is warranted. Cholecystocolonic fistulas are uncommon and when present is usually associated with significant biliary disease. This paper aims to present an evenly atypical manifestation of this variant and much more that it presents in a very rare manner in the form of lower GI Bleeding.

Methods We present a case of a 61-year-old male with Acute Myelogenous Leukaemia with no established biliary disease. $\mathrm{He}$ was post-appendectomy with loop ileostomy. He complained of recurrent abdominal pain with hematochezia.

Results Plain film of the abdomen revealed gas distended bowel loops in the disorganised pattern. Initial colonoscopy prior to the contemplated takedown of ileostomy was done revealing an ascending colon stricture and colitis. Anaemia and infection were addressed. The abdominal pain persisted, and he had massive hematochezia, 2nd look colonoscopy revealed an ulcerated area oozing with blood and bile flowing into the ascending colon hence a fistula was suspected. The patient did not respond to conservative management of the bleeding hence he underwent Right hemicolectomy and cholecystectomy with adhesiolysis and takedown of ileostomy which confirmed the diagnosis of cholecystocolonic fistula and was further supported by the histopathologic findings.

The patient improved post-operatively and was discharged subsequently.

Conclusions Be prudent in handling atypical scenarios in a morbid and complicated patient. A stepwise approach and a high index of suspicion especially in unusual situations can often lead us to the correct diagnosis.

\section{IDDF2018-ABS-0255 IMPACT OF COMMENSAL HUMAN GUT MICROBIOTA IN THE EMERGENCE OF EXTENSIVE DRUG-RESISTANT PATHOGENS}

${ }^{1}$ Sayantan Banerjee*, ${ }^{2}$ Satyabrata Bag, ${ }^{2}$ Jyoti Verma, ${ }^{2} \mathrm{D}$ Anbumani, ${ }^{2}$ Bhabatosh Das. ${ }^{1} \mathrm{All}$ India Institute of Medical Sciences, New Delhi, India; ${ }^{2}$ Centre for Human Microbial Ecology, Translational Health Science and Technology Institute, NCR Biotech Science Cluster, Faridabad, India

\subsection{6/gutjnl-2018-IDDFabstracts.43}

Background Antimicrobial resistance (AMR) in human health associated bacterial pathogens is a serious concern across the globe. Human gastrointestinal tract (GIT) is populated with trillions of microbial species that contribute to nutrient assimilation, host and microbial cell signalling, pathogenesis and antimicrobial resistance (AMR). Indiscriminate usages of antimicrobial in healthcare, livestock, and agriculture provide an evolutionary advantage to the resistant variants. The ascendency of resistant variants threatens the efficacy of most, if not all, of the antimicrobial drugs commonly used to prevent or cure microbial infections. The aim of this current study was to understand the impact of commensal gut microbiota in the emergence of extensively drug-resistant (XDR) enteric pathogens.

Methods Commensal anaerobic gut microbiota was isolated from the healthy Indians without a history of hospitalisation and antibiotic intake in last one year. AMR phenotypes were characterised in an anaerobic workstation using commercially avail Epsilometer test (E-test) strip. Genome of all the isolates was sequenced in GS-FLX + pyrosequencer. Genome assembly was done in GS de novo assembler. Genome annotation and analysis of resistance genes were done using Rapid Annotations using Subsystems Technology (RAST) platform.

\section{Results}

i. AMR traits in enteric bacteria, both in commensals and pathogens, are physically linked with mobile genetic elements (MGEs) and could rapidly disseminate to the bacterial species through horizontal gene transfer (HGT).

ii. Among commensal gut microbiota, the highest number of resistance phenotypes $(n=12)$ and AMR encoding functions were detected in Faecalibacterium prausnitzii.

iii. Among pathogens, Vibrio cholerae and Klebsiella pneumoniae showed maximum resistance phenotypes $(n=22)$.

iv. Genes encoding antibiotic resistance in commensal and pathogenic bacteria are physically linked with MGEs and could disseminate vertically to the progeny and laterally to the distantly related microbial species.

Conclusions XDR pathogenic and commensal enteric bacteria are isolated from healthy Indian subjects without a significant history of hospitalisation or antibiotic consumption. Consequently, the transmissible AMR genes present in the genome of gut commensals could be a potential source of resistance functions for the enteric pathogens.

\section{IDDF2018-ABS-0264 PREVALENCE OF ANXIETY, DEPRESSION AND QUALITY OF LIFE IN AMONG SCHOOL GOING ADOLESCENT WITH IRRITABLE BOWEL SYNDROME}

Nitin Kumar*. Department of Basic and Applied Biology, Vivekananda Global University, Jaipur, Rajasthan, India

\subsection{6/gutjnl-2018-IDDFabstracts.44}

Background Irritable bowel syndrome (IBS) is the most prevalent functional gastrointestinal disorders (FGIDs) that affects different aspects of life and patients experienced depression and anxiety more than others. There have been few Asian studies regarding anxiety and depression associated IBS. The aim of this study was to evaluate the frequency, magnitude and importance of anxiety and depression in among school going adolescent with IBS in Jaipur city, India.

Methods A Cross-sectional survey of students of four classes from 9th to 12th studying in government schools in Jaipur city, India. Ten government schools in Jaipur were randomly selected through lottery method. In each school, for each of the four classes, a section was randomly selected again by the lottery method. Forty students were selected from each school reaching a sample size of 470 . This clinical trial study was done in IBS patients (with mild-to-moderate symptoms) divided into two case and control groups. All participants were asked to complete self-administered questionnaires: one addressing symptom severity and the hospital anxiety and depression scale (HADS). The patients were also asked to complete the IBS-specific quality of life (IBS-QOL) questionnaire.

Results Anxiety and depression were observed in $32.1 \%$ and $34.5 \%$ of IBS patients, respectively, and in $26.6 \%$ and $17.2 \%$ of healthy subjects, respectively $(\mathrm{p}<0.05$ for both) in school adolescent. Both anxiety and depression were associated with self-reported symptom severity $(\mathrm{p}<0.05$ and $\mathrm{p}<0.05$, respectively). As determined by multivariate analysis, symptom severity was the most important factor in the prediction of anxiety and depression. Self-reported symptom severity and 